This item was submitted to Loughborough's Research Repository by the author.

Items in Figshare are protected by copyright, with all rights reserved, unless otherwise indicated.

\title{
Normal modes of a quasi-one-dimensional multichain complex plasma
}

PLEASE CITE THE PUBLISHED VERSION

http://dx.doi.org/10.1103/PhysRevE.70.036406

\section{PUBLISHER}

(C) The American Physical Society

VERSION

VoR (Version of Record)

LICENCE

CC BY-NC-ND 4.0

REPOSITORY RECORD

Piacente, G., F.M. Peeters, and Joseph J. Betouras. 2019. "Normal Modes of a Quasi-one-dimensional Multichain Complex Plasma". figshare. https://hdl.handle.net/2134/12773. 


\title{
Normal modes of a quasi-one-dimensional multichain complex plasma
}

\author{
G. Piacente, ${ }^{1}$ F. M. Peeters, ${ }^{1}$ and J. J. Betouras ${ }^{1,2}$ \\ ${ }^{1}$ Department of Physics, University of Antwerp (Campus Drie Eiken), B-2610 Antwerpen, Belgium \\ ${ }^{2}$ Max Planck Institute for the Physics of Complex Systems, Noethnitzer Strasse 38, Dresden 01187, Germany
}

(Received 23 April 2004; published 21 September 2004)

\begin{abstract}
We studied equally charged particles, suspended in a complex plasma, which move in a plane and interact with a screened Coulomb potential (Yukawa type) and with an additional external confining parabolic potential in one direction, which makes the system quasi-one-dimensional (Q1D). The normal modes of the system are studied in the presence of dissipation. We also investigated how a perpendicular magnetic field couples the phonon modes with each other. Two different ways of exciting the normal modes are discussed: (1) a uniform excitation of the Q1D lattice, and (2) a local forced excitation of the system in which one particle is driven by, e.g., a laser. Our results are in very good agreement with recent experimental findings on a finite single chain system [Liu et al., Phys. Rev. Lett. 91, 255003 (2003)]. Predictions are made for the normal modes of multichain structures in the presence of damping.
\end{abstract}

DOI: 10.1103/PhysRevE.70.036406

PACS number(s): 52.27.Lw, 63.20.Dj

\section{INTRODUCTION}

Since the first experimental observations of the formation of Coulomb quasilattices [1] involving highly charged dust particles in 1994 [2-4], the research field of complex plasmas has seen sustained growth. Complex plasmas consist of micrometer-sized ("dust") particles immersed in a gaseous plasma background. Dust particles typically acquire a negative charge of several thousand elementary charges, so they interact with each other through their strong electrostatic repulsion. In the experiment, particles are trapped in a horizontal layer by a shallow parabolic well, due to two electrodes, and can be suspended in a sheath above the electrodes, where the gravity force is balanced by the electrostatic force. When the electrostatic energy of neighboring particles exceeds the thermal energy by an amount $\Gamma$, the particles arrange themselves in regular, solidlike structures, i.e., Wigner crystals [5]. The mutual Coulomb repulsion of the dust grains is partly screened by the polarization of the surrounding plasma particles, mostly by the gas ions which represent the major species in the sheath. Therefore, the average interparticle potential can be well represented by a Yukawa (Debye-Hückel) potential [6].

Complex plasmas provide an additional system for the study of classical crystalline and liquid dynamics and the melting processes. For particle size of the order of micrometers the dynamical behavior can be monitored directly with the use of optical microscopes [1].

In the present work we study thoroughly the normal modes of a classical quasi-one-dimensional (Q1D) multichain complex plasma. Such a Q1D system was recently realized experimentally by giving a proper shape to the electrodes $[7,8]$. Experimentally, many other quasi-onedimensional or strictly one-dimensional systems have been realized over the years. Colloidal particles, suspended in aqueous solution, can be trapped in a potential well created by two counterpropagating laser beams which form a onedimensional coupled array [9]. A Coulomb chain confined in a storage ring [10], as well as ordered electrons on microchannels filled by liquid helium [11], are other examples of
Q1D classical systems. The latter is one of the candidates to be used for quantum computing $[12,13]$. On the atomic scale a chainlike system can be found in compounds such as $\mathrm{Hg}_{3-\delta} \mathrm{AsF}_{6}$ [14] and in low dimensional systems formed on surfaces [15]. A one-dimensional chain of gas atoms adsorbed by carbon nanotubes has been produced in a laboratory and its phonon spectrum has been calculated theoretically assuming a Lennard-Jones interaction potential $[16,17]$.

The classical model we propose in the present paper reveals a nontrivial phase diagram at zero temperature and allows us to calculate dispersion relations for the normal modes, which can be directly investigated experimentally. Several generic aspects of the model were investigated recently [18]. Here, the main focus is on the normal modes of the system and how they depend on different physical situations, e.g., frictional forces and the way they are excited. We will make connection with recent experimental work $[7,19]$.

The structure of the paper is as follows. We first give in Sec. II an overview of the model, stressing the ground state properties. In Sec. III we present what has been known so far for the normal modes of the system, adding more results for clarification, and then we turn to additional results with respect to the presence of the gas drag (friction) with or without an applied magnetic field. Finally, we turn our attention to the forced oscillations in Sec. V, where we discuss recent experiments on normal modes in single chain systems. Predictions for multichain configurations are presented in Sec. VI. Finally we conclude in Sec. VII.

\section{MODEL AND GENERAL PROPERTIES}

We consider a system of equally charged particles with coordinates $\vec{r}_{i}=\left(x_{i}, y_{i}\right)$ moving in a plane and interacting with each other through a Yukawa-type potential (the screening length $\lambda$ is an external parameter which is measured in the experiment [20]) and are confined by a parabolic potential in the $y$ direction. The dimensionless Hamiltonian of the system is given by 


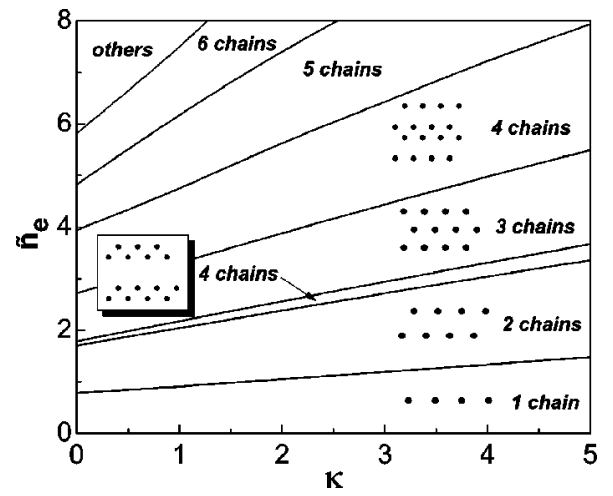

FIG. 1. The $T=0$ structural phase diagram as a function of the inverse screening length $\kappa$ and the density $\widetilde{n}_{e}$. The plotted quantities are dimensionless, as for all the figures in the paper.

$$
H^{\prime}=\sum_{i \neq j} \frac{\exp \left(-\kappa\left|\vec{r}_{i}^{\prime}-\vec{r}_{j}^{\prime}\right|\right)}{\left|\vec{r}_{i}^{\prime}-\vec{r}_{j}^{\prime}\right|}+\sum_{i} y_{i}^{\prime 2},
$$

where $H^{\prime}=H / E_{0}, \quad \kappa=r_{0} / \lambda$, and $\vec{r}^{\prime}=\vec{r} / r_{0}$, with $r_{0}$ $=\left(2 q^{2} / m \varepsilon \omega_{0}^{2}\right)^{1 / 3}$ as the unit of length and $E_{0}$ $=\left(m \omega_{0}^{2} q^{4} / 2 \varepsilon^{2}\right)^{1 / 3}$ as the unit of energy; $m$ and $q$ are the mass and the charge of the particles, respectively, $\varepsilon$ is the dielectric constant of the medium the particles are moving in, and $\omega_{0}$ measures the strength of the confining potential. The dimensionless time is defined as $t^{\prime}=\omega_{0} t$. Finally, it is possible to define a dimensionless temperature as $T^{\prime}=T / T_{0}$ with $T_{0}$ $=E_{0} / k_{B}=\left(m \omega_{0}^{2} q^{4} / 2 \varepsilon^{2}\right)^{1 / 3} k_{B}^{-1}$.

In our previous work [18] we investigated the ground state and the melting of this Q1D system. We summarize here the main results, which we will need in the next sections. At $T=0$ the particles crystallize in a chainlike crystal structure, with a linear density equally distributed among the chains. In the case of multiple chains, if $a$ is the separation between two neighboring particles in the same chain, the chains are staggered by $a / 2$ in the $x$ direction, because this arrangement minimizes the electrostatic repulsion. The results for the ground state configuration are summarized in the phase diagram depicted in Fig. 1.

The dimensionless linear density is defined as $\tilde{n}_{e}=\nu r_{0} / a$, where $\nu$ is the number of chains. For low densities the particles crystallize in a single chain; with increasing density a continuous transition ("zigzag" [22]) occurs and the single chain splits into two chains. On further increasing the density we found the remarkable behavior that the four-chain structure is stabilized before the three-chain structure. The $2 \rightarrow 4$ chain transition occurs through a "zigzag" transition of each of the chains accompanied by a shift of $a / 4$ along $x$. This four-chain configuration has a relatively small stability range after which it transits to a three-chain configuration through a discontinuous, i.e., first order, phase transition. For higher values of the density, the four-chain configuration again attains the lowest energy. A further increase of $\tilde{n}_{e}$ will lead to more chains, that is six, seven, and so on. The structural transitions are discontinuous, i.e., first order, except for the $1 \rightarrow 2$ transition.
On raising the temperature the ordered structure melts. We studied in detail the melting for this kind of system in Ref. [18]. Due to the anisotropy in the two directions, a different behavior of the system in the $x$ and $y$ directions was found. Two different melting temperatures $T_{x}$ and $T_{y}$ can be assigned. The main features of the transition from the ordered state to the liquid state are (i) a reentrant behavior as a function of density; (ii) a region in density for which the system melts first in the unconfined direction and then in the confined one: this regime resembles the findings of Ref. [21] in the floating solid regime; (iii) reentrant melting occurring near the structural transition points. For the nearly Coulomb limit $(\kappa=0.01)$ there is no evidence of anisotropic melting, that is, the system behaves more isotropically. Furthermore, the Coulomb system has a melting temperature which is on average 15-20\% higher than for the screened Coulomb interparticle interaction with $\kappa=1$. On the other hand, for higher values of $\kappa$, the system behaves more anisotropically and the difference between $T_{x}$ and $T_{y}$ is enhanced. In the case $\kappa=3$ the melting temperature is on average $10-15 \%$ lower than for the case $\kappa=1$.

\section{NORMAL MODES}

In the present paper we are interested in the normal modes of the chain structures, and in particular how these modes are modified in the presence of frictional forces. We will consider (1) the uniformly damped motion of a normal mode, and (2) the damped propagation of a local forced oscillation of a single particle. For these purposes we review briefly the normal modes in the absence of friction.

\section{A. Dispersion relations in the absence of friction}

In the absence of drag due to the ion gas and exploiting the standard harmonic approximation, the equations of motion for small oscillations about the lattice equilibrium positions in dimensionless units are in the single chain case

$$
\begin{gathered}
\frac{d^{2} x_{i}^{\prime}}{d t^{\prime 2}}=-\left.\frac{1}{2} \sum_{j} \frac{\partial^{2} U}{\partial x_{i}^{\prime} \partial x_{j}^{\prime}}\right|_{e q} x_{j}^{\prime}-\left.\frac{1}{2} \sum_{j} \frac{\partial^{2} U}{\partial x_{i}^{\prime} \partial y_{j}^{\prime}}\right|_{e q} y_{j}^{\prime}, \\
\frac{d^{2} y_{i}^{\prime}}{d t^{\prime 2}}=-\left.\frac{1}{2} \sum_{j} \frac{\partial^{2} U}{\partial y_{i}^{\prime} \partial x_{j}^{\prime}}\right|_{e q} x_{j}^{\prime}-\left.\frac{1}{2} \sum_{j} \frac{\partial^{2} U}{\partial y_{i}^{\prime} \partial y_{j}^{\prime}}\right|_{e q} y_{j}^{\prime}-y_{i}^{\prime},
\end{gathered}
$$

where $U=\exp \left(-\kappa\left|\vec{r}_{i}^{\prime}-\vec{r}_{j}^{\prime}\right|\right) /\left|\vec{r}_{i}^{\prime}-\vec{r}_{j}^{\prime}\right|$ is the interparticle interaction potential. Considering the translational invariance of the system along the $x$ direction, we search for solutions in the form

$$
\left(x_{n}^{\prime}, y_{n}^{\prime}\right) \propto \exp [i(k n a-\omega t)]
$$

which results in

$$
\left[\left(\omega^{2}-\delta_{\beta y}\right) \delta_{\alpha \beta, i j}-D_{\alpha \beta, i j}\right] Q_{\beta, j}=0
$$


where $D_{\alpha \beta, i j}$ is the dynamical matrix, that is, the matrix of the second derivatives of the Yukawa potential, calculated at the equilibrium configuration; $Q_{\beta, j}$ is the displacement of particle $j$ from its equilibrium position in the $\beta$ direction; $(\alpha, \beta) \equiv(x, y), \delta_{\alpha \beta, i j}, \delta_{i j}$, and $\delta_{\beta y}$ are unit matrices; in particular $\delta_{\beta y}$ takes into account the effect of the confining potential. All the frequencies are measured in units of $\omega_{0}$.

The number of chains determines the number of particles in each unit cell and therefore the number of degrees of freedom per unit cell. So if $l$ is the number of chains there will be $2 l$ branches for the normal mode dispersion curves. Note that for ordinary bidimensional crystals there are two acoustical branches and $2 p-2$ optical branches [23], if $p$ is the number of atomic species in the unit cell.

Solving Eq. (4) explicitly for the single chain configuration, we obtain that the acoustical and optical eigenfrequencies are given respectively by:

$$
\begin{aligned}
\omega_{a c}(k)= & {\left[\tilde{n}_{e}^{3} \sum_{j=1}^{\infty} \frac{\exp \left(-j \kappa / \widetilde{n}_{e}\right)}{j^{3}}\left(2+\frac{2 j \kappa}{\widetilde{n}_{e}}+\frac{j^{2} \kappa^{2}}{\tilde{n}_{e}^{2}}\right)\right.} \\
& \times[1-\cos (k a j)]]^{1 / 2}, \\
\omega_{\text {opt }}(k)= & {\left[1-\widetilde{n}_{e}^{3} \sum_{j=1}^{\infty} \frac{\exp \left(-j \kappa / \widetilde{n}_{e}\right)}{j^{3}}\left(1+\frac{j \kappa}{\widetilde{n}_{e}}\right)\right.} \\
& \times[1-\cos (k a j)]]^{1 / 2},
\end{aligned}
$$

where $k$ is the wave number. Numerical results for the dispersion relations were presented in Ref. [18].

It is interesting to notice that for the acoustical branch the dispersion is positive, that is, the phase and group velocities have the same sign, while for the optical branch the dispersion is negative, i.e., the group velocity is negative. Physically, the negative dispersion for the single chain optical branch can be understood by considering that the electrostatic repulsion acts oppositely to the force of the confining potential and this reduces the oscillation frequency with increasing $k$.

Another notable feature is the softening of the optical branch, accompanied by a hardening of the acoustical branch at the values of $\tilde{n}_{e}$ and $\kappa$ where the $1 \rightarrow 2$ structural transition is observed (see Fig. 8 of Ref. [18]), which confirms that 1 $\rightarrow 2$ is a continuous transition.

When a magnetic field is applied in the perpendicular direction to the plane in which the particles are moving, the equations of motions are modified and $\dot{y}_{i}^{\prime} \omega_{c}^{\prime}$ is added to the right hand side of Eq. (2a) and $-\dot{x}_{i}^{\prime} \omega_{c}^{\prime}$ to the right hand side of Eq. (2b), where $\vec{\omega}_{c}=q \vec{B} / m c$ is the cyclotron frequency and $\omega_{c}^{\prime}=\omega_{c} / \omega_{0}$. It is known [24] that in a classical system an external magnetic field does not alter the statistical properties of the system and consequently the structural properties should be insensitive to the magnetic field strength. On the other hand, the character of motion of the particles is altered significantly because now the $x$ and $y$ motions are coupled. The spectrum of an infinite bidimensional crystal in a mag- netic field was obtained in Refs. [25,26]. Following Ref. [26], the dispersion relation for our Q1D system in the presence of a perpendicular magnetic field $B$ is obtained from

$$
\left[\left(\omega^{2}-\delta_{\beta y}\right) \delta_{\alpha \beta, i j}-D_{\alpha \beta, i j}+i \omega \omega_{c} \xi_{\alpha \beta} \delta_{i j}\right] Q_{\beta, j}=0 .
$$

\section{B. Dispersion relations in the presence of friction}

In laboratory experiments on a dusty plasma the particles experience a frictional drag due mainly to the background neutral gas as well as ions. This drag has a significant effect on the dispersion curves of the normal modes. In order to compare experimental data with theory, it is necessary to develop a theoretical model in which the structure of the crystal as well as damping are included as essential elements. This can be easily done by adding explicitly the friction term in the equations of motion. For the single chain configuration $\gamma^{\prime} \dot{x}_{i}^{\prime}$ should be added to the left hand side of Eq. (2a) and $\gamma^{\prime} \dot{y}_{i}^{\prime}$ to the left hand side of Eq. (2b), where $\gamma^{\prime}=\gamma / \omega_{0}$ is the dimensionless frictional drag coefficient. Similar equations hold naturally in multichain structures. The equations of motion for the two- and three-chain structures are reported for completeness in Appendix A.

Proceeding as before, in this case the eigenfrequencies are determined by

$$
\left[\left(\omega^{2}-\delta_{\beta y}+i \gamma \omega\right) \delta_{\alpha \beta, i j}-D_{\alpha \beta, i j}\right] Q_{\beta, j}=0 .
$$

For a single chain Eq. (7) gives explicitly:

$$
\begin{aligned}
\omega_{a c}^{2}+ & i \gamma \omega_{a c}-\tilde{n}_{e}^{3} \sum_{j=1}^{\infty} \frac{\exp \left(-j \kappa / \tilde{n}_{e}\right)}{j^{3}}\left(2+\frac{2 j \kappa}{\widetilde{n}_{e}}+\frac{j^{2} \kappa^{2}}{\widetilde{n}_{e}^{2}}\right) \\
\times & {[1-\cos (k a j)]=0, } \\
\omega_{o p t}^{2} & +i \gamma \omega_{o p t}-1+\widetilde{n}_{e}^{3} \sum_{j=1}^{\infty} \frac{\exp \left(-j \kappa / \widetilde{n}_{e}\right)}{j^{3}}\left(1+\frac{j \kappa}{\widetilde{n}_{e}}\right) \\
\times[1-\cos (k a j)] & =0,
\end{aligned}
$$

from which we obtain the solutions

$$
\begin{aligned}
\omega_{a c}(k)= & {\left[\tilde{n}_{e}^{3} \sum_{j=1}^{\infty} \frac{\exp \left(-j \kappa / \widetilde{n}_{e}\right)}{j^{3}}\left(2+\frac{2 j \kappa}{\tilde{n}_{e}}+\frac{j^{2} \kappa^{2}}{\tilde{n}_{e}^{2}}\right)\right.} \\
\times & \left.\times[1-\cos (k a j)]-\frac{\gamma^{2}}{4}\right]^{1 / 2}-i \frac{\gamma}{2}, \\
\omega_{o p t}(k)= & {\left[1-\widetilde{n}_{e}^{3} \sum_{j=1}^{\infty} \frac{\exp \left(-j \kappa / \tilde{n}_{e}\right)}{j^{3}}\left(1+\frac{j \kappa}{\widetilde{n}_{e}}\right)\right.} \\
& \left.\times[1-\cos (k a j)]-\frac{\gamma^{2}}{4}\right]^{1 / 2}-i \frac{\gamma}{2} .
\end{aligned}
$$

The analytical expression for the two- and three-chain eigenfrequencies are reported in Appendix B.

In the limit of small wave number $k$, we find for $\kappa / \tilde{n}_{e}$ $\gg 1$ that Eqs. (9a) and (9b) reduce, respectively, to 


$$
\begin{gathered}
\omega_{a c}(k)=\left[e^{-\kappa / \tilde{n}_{e}} \frac{\kappa^{2} \tilde{n}_{e}}{2}\left(1-\frac{k^{2} a^{2}}{12}\right) k^{2} a^{2}-\frac{\gamma^{2}}{4}\right]^{1 / 2}-i \frac{\gamma}{2}, \\
\omega_{o p t}(k)=\left[1-e^{-\kappa / \tilde{n}_{e}} \frac{\tilde{n}_{e}^{2} \kappa}{2}\left(1-\frac{k^{2} a^{2}}{12}\right) k^{2} a^{2}-\frac{\gamma^{2}}{4}\right]^{1 / 2}-i \frac{\gamma}{2},
\end{gathered}
$$

while for $\kappa / \tilde{n}_{e} \ll 1$ we find

$$
\begin{aligned}
\omega_{a c}(k)= & \left\{\left[\frac{3}{2}+\ln \left(\frac{\tilde{n}_{e}}{\kappa}\right)-\frac{\tilde{n}_{e}}{12}\left(1+\frac{5 \tilde{n}_{e}}{12}\right) k^{2} a^{2}\right] \tilde{n}_{e}^{3} k^{2} a^{2}-\frac{\gamma^{2}}{4}\right\}^{1 / 2} \\
& -i \frac{\gamma}{2}, \\
\omega_{\text {opt }}(k)= & \left\{1-\left[1+\ln \left(\frac{\tilde{n}_{e}}{\kappa}\right)-\frac{\tilde{n}_{e}}{\kappa}\left(1+\frac{2 \tilde{n}_{e}}{\kappa}\right) k^{2} a^{2}\right]\right. \\
& \left.\times \frac{\tilde{n}_{e}^{3}}{2} \frac{k^{2} a^{2}}{12}-\frac{\gamma^{2}}{4}\right\}^{1 / 2}-i \frac{\gamma}{2} .
\end{aligned}
$$

The real part of the frequency corresponds to the oscillatory motion while the damping in the time domain is given by the imaginary term $i \gamma / 2$. Note that friction also affects the value of the frequencies of normal modes. In Fig. 2 the normal mode spectra are reported for different configurations of the system for different values of $\widetilde{n}_{e}, \kappa$, and $\gamma^{\prime}$. We used in our calculations values for the parameters inferred from the experiment [7]. The behavior of the dispersion curves reflects rather closely the case without damping. Some additional features should, however, be stressed: (i) the effect of friction results in general in a reduction of the frequencies of vibration both for the longitudinal motion and for the transverse one; (ii) for very small values of the wave number the acoustical vibrations cannot be excited, which implies that they are overdamped; such waves can be excited only when $k>k^{*}\left(\widetilde{n}_{e}, \kappa, \gamma^{\prime}\right)$; (iii) the softening of the optical mode at the critical density $\widetilde{n}_{e}^{*}$ for the transition $1 \rightarrow 2$ depends on $\gamma^{\prime}$; in particular, the presence of friction reduces the value of $\widetilde{n}_{e}^{*}$ [see Fig. 2(b)].

When we include a magnetic field the damped normal modes are determined by

$$
\left[\left(\omega^{2}-\delta_{\beta y}+i \gamma \omega\right) \delta_{\alpha \beta, i j}-D_{\alpha \beta, i j}+i \omega \omega_{c} \xi_{\alpha \beta} \delta_{i j}\right] Q_{\beta, j}=0 .
$$

The corresponding dispersion curves for the single and multichain structures are reported in Fig. 3. The behavior of the curves resembles the case without damping but with an additional shift in frequency due to friction. Note that in this case it is no longer possible to obtain the phonon frequencies analytically. The anticrossing between the two branches in the one-chain configuration is still present [see Fig. 3(b)], as in the case without friction (see Fig. 11 of Ref. [18]). It is remarkable that the cyclotron motion and the friction are coupled and the magnetic field introduces a dispersion in the imaginary part of $\omega$ as well. Now, $\operatorname{Im}(\omega)$ is no longer constant as a function of the wave vector as in the case without

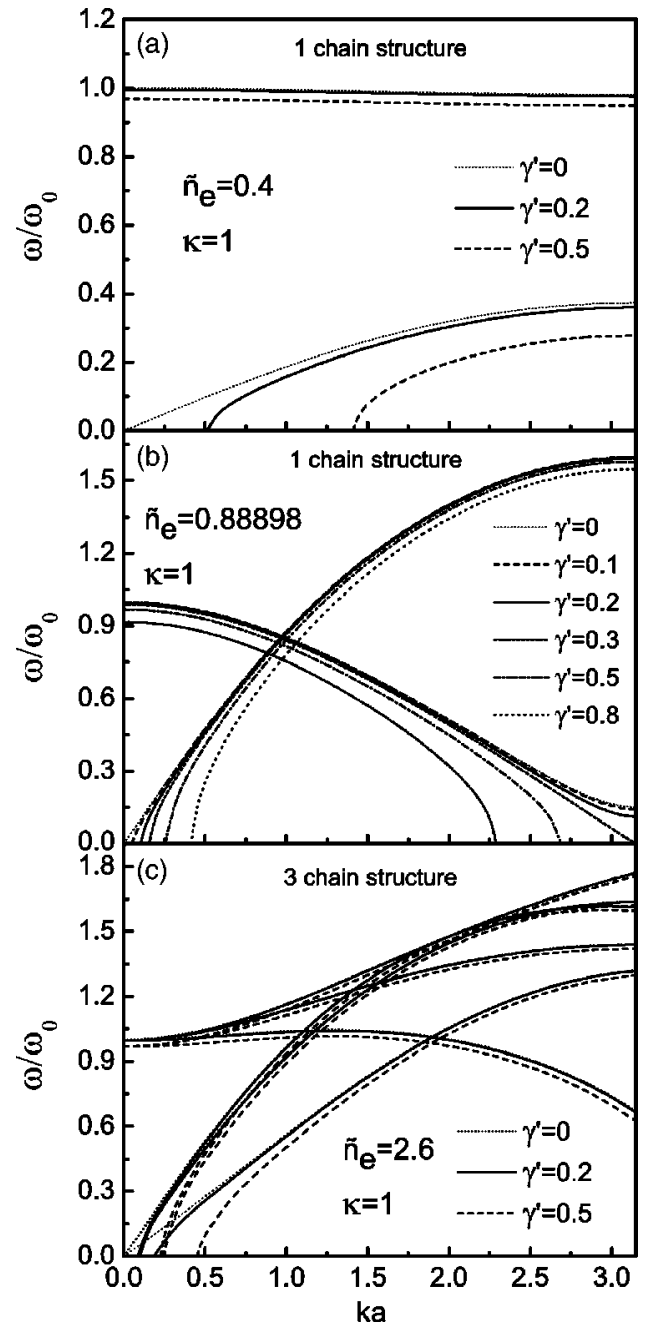

FIG. 2. Dispersion curves for the normal modes in the presence of friction for different values of parameters for a one- (a), and three- (c) chain structure. Dependence of the acoustical and optical branches in the one-chain case on the friction coefficient at the critical density where the softening of the optical mode is observed is given in (b).

a magnetic field. Friction mainly alters the acoustical branches of the magnetophonon modes for small wave vectors.

\section{FORCED OSCILLATIONS IN A SINGLE CHAIN STRUCTURE}

In the experiment of Refs. [7,28-31,33] the system is set into oscillation by an external driving force which acts on the system continuously. The frequency of such a forced oscillation is then determined by the frequency of the driving force and not by the resonant frequencies. This is the effective situation in experiments where particle motions are excited by laser manipulation, which makes it possible to excite and test the dispersion relations of certain types of lattice wave [27-30]; these are longitudinal waves and, most recently, transverse waves were also observed [7,31,32]. Laser light exerts a radiation pressure on the particles with a magnitude 


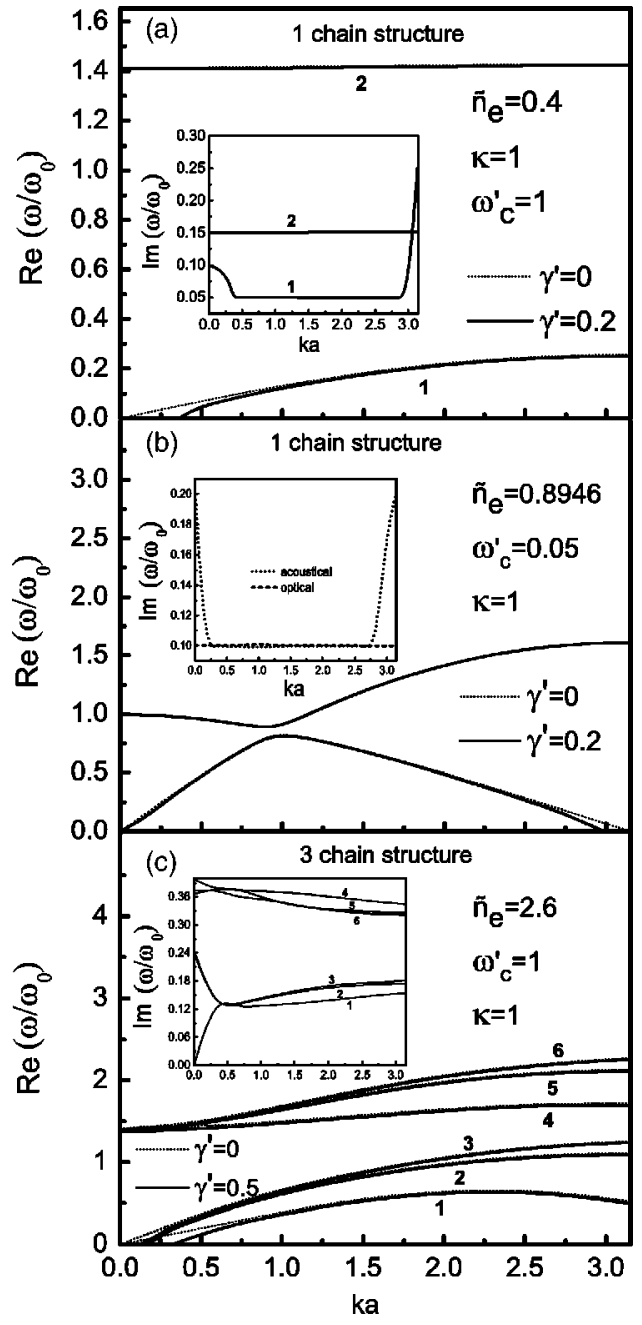

FIG. 3. The same as Fig. 2 but now a magnetic field of strength $\omega_{c} / \omega_{0}=1$ is present. The insets depict the damping of magnetophonon modes.

proportional to the laser intensity [33]. In these cases the frequency is purely real since the modes are driven. What is observed in the experiments is that as the wave propagates it is spatially damped, which can be interpreted in term of a complex wave number [27] $k=k_{r}+i k_{i}$. Following this idea and considering that excitations take place when the driving frequency is close to the free frequency of the modes, we may neglect to first approximation for the single chain structure the external force, and we look for particular solutions of the equations of motion in the form

$$
\left(x_{n}^{\prime}, y_{n}^{\prime}\right) \propto \exp \left[i\left(k_{r} n a-\omega t\right)\right] \exp \left(-k_{i} n a\right)
$$

as was done in Ref. [7] for theoretical calculation of the optical branch. This yields for the acoustical and optical branches, respectively,

$$
\begin{aligned}
\omega_{a c}^{2} & +i \gamma \omega_{a c}-\tilde{n}_{e}^{3} \sum_{j=1}^{\infty} \frac{\exp \left(-j \kappa / \tilde{n}_{e}\right)}{j^{3}}\left(2+\frac{2 j \kappa}{\tilde{n}_{e}}+\frac{j^{2} \kappa^{2}}{\tilde{n}_{e}^{2}}\right) \\
& \times\left[1-\cos \left(k_{r} a j\right) \cosh \left(k_{i} a j\right)+i \sin \left(k_{r} a j\right) \sinh \left(k_{i} a j\right)\right]=0,
\end{aligned}
$$
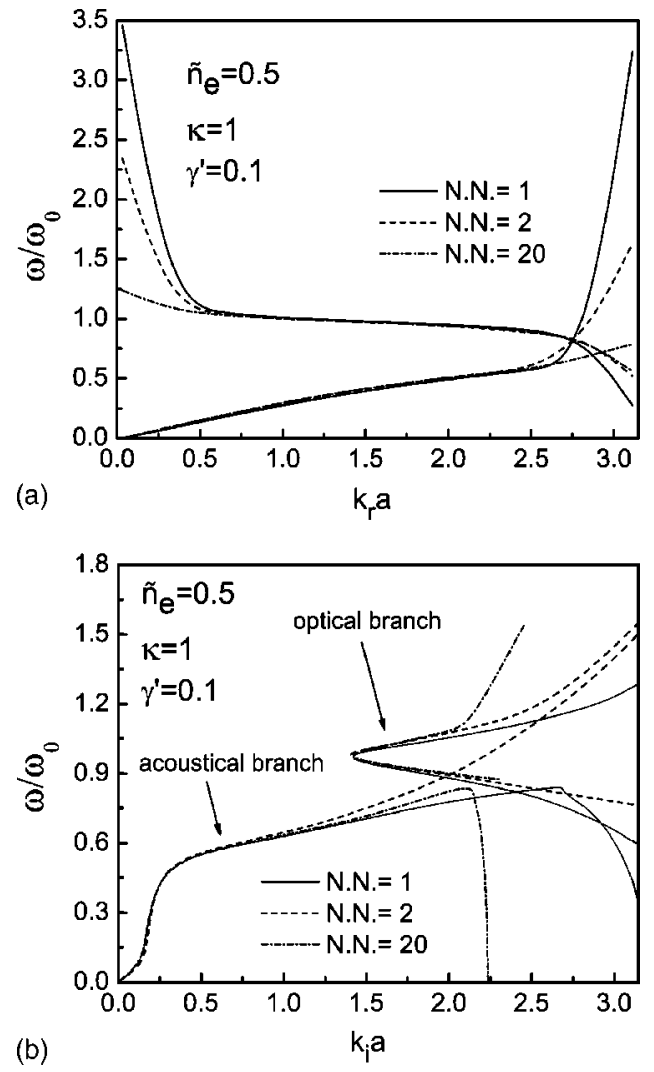

FIG. 4. Dispersion relations for a single chain structure as a function of the (a) real part and (b) imaginary part of the wave vector. The curves show a strong dependence on the number of nearest neighbors included in the calculations (N.N.), at the edges of the first Brillouin zone.

$$
\begin{aligned}
\omega_{o p t}^{2} & +i \gamma \omega_{o p t}-1+\widetilde{n}_{e}^{3} \sum_{j=1}^{\infty} \frac{\exp \left(-j \kappa / \widetilde{n}_{e}\right)}{j^{3}}\left(1+\frac{j \kappa}{\widetilde{n}_{e}}\right) \\
\times & \times\left[1-\cos \left(k_{r} a j\right) \cosh \left(k_{i} a j\right)+i \sin \left(k_{r} a j\right) \sinh \left(k_{i} a j\right)\right]=0 .
\end{aligned}
$$

Requiring the frequency $\omega$ to be real, the two equations generate a system of two nonlinear equations, for $k_{r}$ and $k_{i}$. The results of the calculation are reported in Fig. 4. This approach has some limits, however. First of all, once the laser acts on a specific particle [7], it is no longer possible to consider all the particle as identical, i.e., the presence of an external force breaks the symmetry of the system which is taken into account in Eq. (13) by considering the driven particle as being at $x_{n=0}=0$. Second, the convergence of the series in Eq. (14a) and (14b) is no longer guaranteed. The condition that must be satisfied in order to have a convergent sum is $k_{i} a \leqslant \kappa / \tilde{n}_{e}$. As seen from Fig. 4(b), this condition is not always satisfied (in the specific case considered in the picture the condition for convergence is $k_{i} a \leqslant 2$ ). Note that the dispersion curves depend strongly on the number of terms considered in the sum at the edges of the first Brillouin zone [Fig. 4(a)], while it is practically independent of the number of neighbors considered in the sum in the middle of the first Brillouin zone. The system of equations arising from Eqs. (14a) and (14b) is not defined when $k a=0$ and $k a=\pi$, 
because in this case the equation for the imaginary part is identically zero. This clearly shows the inapplicability of this approach to obtain the phonon spectrum in the presence of friction.

The reason for the divergence of the sums in Eqs. (14a) and (14b) is a consequence of the fact that the last exponent in Eq. (13) blows up for negative values of $n$. This would suggest that alternatively we should look for solutions of the equation of motion in the form

$$
\left(x_{n}^{\prime}, y_{n}^{\prime}\right) \propto \exp \left[i\left(k_{r} n a-\omega t\right)\right] \exp \left(-k_{i}|n| a\right),
$$

i.e., damped waves propagating from the location of the external excitation. But in this case in the imaginary part in Eqs. (14a) and (14b) the hyperbolic sine term is replaced by $\exp \left(-k_{i}|j| a\right)$ and consequently the sum gives zero. As a result, Eqs. (14a) and (14b) do not have any real solutions for the phonon frequency, and this approach also fails.

In order to explain some recent experimental results on the transverse modes of a finite one-dimensional chain [7], excited by striking one particle with two counterpropagating laser beams such that the effective force acting on the particle is $I_{0} \sin \omega t$, with $I_{0}$ the intensity of the beam, we have followed another approach. We first consider a single finite chain of $N$ particles confined in the $y$ direction. On one of the particles a time varying force is acting. We studied the small displacements from the equilibrium configuration of each particle, limiting ourselves to first neighbor interactions, which is valid for $k a>1$. The equations of motion for such a system are

$$
\begin{aligned}
\frac{d^{2} x_{l}^{\prime}}{d t^{\prime 2}}+\gamma^{\prime} \frac{d x_{l}^{\prime}}{d t^{\prime}}= & \tilde{n}_{e}^{3} e^{-\kappa / \tilde{n}_{e}}\left(2+\frac{2 \kappa}{\widetilde{n}_{e}}+\frac{\kappa^{2}}{\widetilde{n}_{e}^{2}}\right)\left(x_{l+1}^{\prime}+x_{l-1}^{\prime}-2 x_{l}^{\prime}\right) \\
& +F_{0}^{x} e^{-i \omega t} \delta_{l, N}, \\
\frac{d^{2} y_{l}^{\prime}}{d t^{\prime 2}}+\gamma^{\prime} \frac{d y_{l}^{\prime}}{d t^{\prime}}= & -\tilde{n}_{e}^{3} e^{-\kappa / \tilde{n}_{e}}\left(1+\frac{j \kappa}{\widetilde{n}_{e}}\right)\left(y_{l+1}^{\prime}+y_{l-1}^{\prime}-2 y_{l}^{\prime}\right)-y_{l}^{\prime} \\
& +F_{0}^{y} e^{-i \omega t} \delta_{l, N / 2}
\end{aligned}
$$

with $l=1,2, \ldots, N$ and $F_{0}^{x, y}$ the dimensionless strength of the driving force. In order to excite the longitudinal vibrations we have considered a force directed along $x$ and acting on one of the extremities of the chain, while to excite the transverse modes the force acts on the particle in the middle of the chain and with $y$ component only, as was done experimentally in Ref. [7]. Looking for a particular solution of Eqs. (15) of the form

$$
\left(x_{l}^{\prime}, y_{l}^{\prime}\right)=\left(A_{l}^{x}, A_{l}^{y}\right) e^{-i \omega t},
$$

we obtained the following set of inhomogeneous linear equations for the displacements $A_{l}$ :

$$
\beta_{1} A_{l-1}^{x}+\left(\omega_{a c}^{2}+i \gamma \omega_{a c}-2 \beta_{1}\right) A_{l}^{x}+\beta_{1} A_{l+1}^{x}-F_{0}^{x} \delta_{l, N}=0,
$$
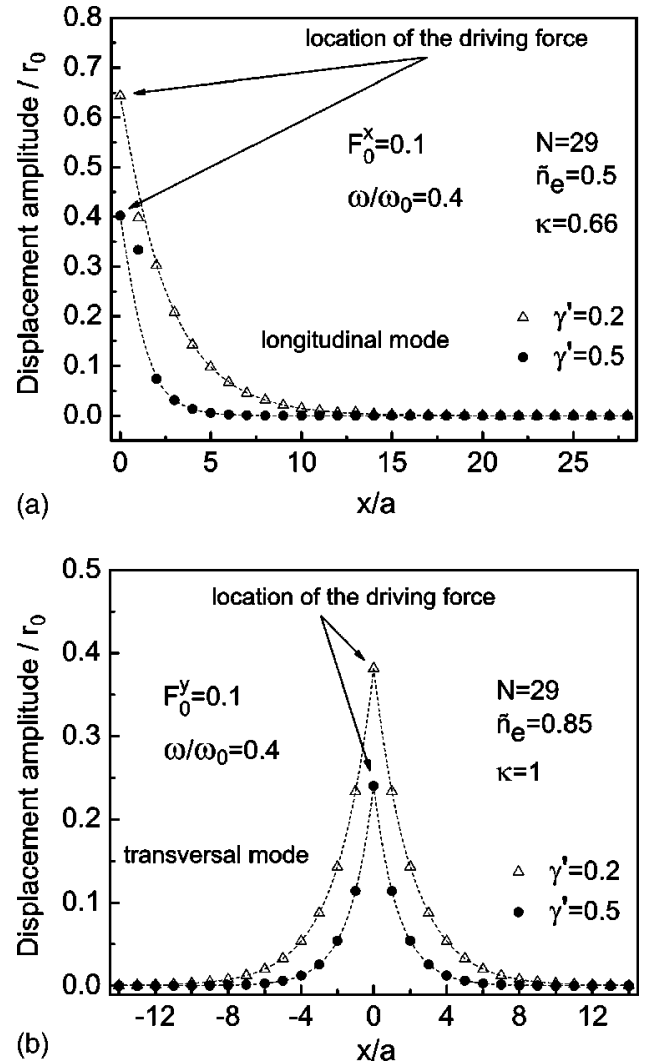

FIG. 5. Amplitude of the displacement of particles as a function of the distance from the location of the driving force: (a) displacements along the $x$ direction when the excited particle is at the extremity of the chain; (b) displacements along the $y$ direction when the excited particle is in the middle of the chain. Exponential fits to the numerical data are shown by the dotted curves.

$$
\beta_{2} A_{l-1}^{y}+\left(\omega_{o p t}^{y}-1+i \gamma \omega_{o p t}-2 \beta_{2}\right) A_{l}^{y}+\beta_{2} A_{l+1}^{y}-F_{0}^{y} \delta_{l, N / 2}=0,
$$

where $\beta_{1}=\widetilde{n}_{e}^{3} e^{-\kappa / \tilde{n}_{e}}\left(2+2 \kappa / \widetilde{n}_{e}+\kappa^{2} / \widetilde{n}_{e}^{2}\right) \quad$ and $\quad \beta_{2}=\widetilde{n}_{e}^{3} e^{-\kappa / \tilde{n}_{e}}(1$ $+\kappa\left(\tilde{n}_{e}\right)$. The solution to these equations may easily be obtained from Kramer's rule [34,35]

$$
A_{l}=\frac{D_{l}(\omega)}{D(\omega)}
$$

where $D(\omega)$ is the determinant of the coefficients of $A_{l}$ in Eqs. (17) and $D_{l}(\omega)$ is the modification in $D(\omega)$ resulting when the $l$ th column is replaced by $\left(F_{0}, 0,0, \ldots, 0\right)$ for the longitudinal motion and $\left(0,0, \ldots, 0, F_{0}, 0, \ldots, 0,0\right)$ for the transverse motion, respectively. $A_{l}$ are complex quantities when $\gamma^{\prime} \neq 0$ and the formalism developed above allows us to calculate amplitudes and phases. The analytical expressions for $A_{l}$ are reported in Appendix C.

In Figs. 5(a) and 5(b) we show the amplitudes of the displacements for the longitudinal and transverse motions as a function of particle position along the chain. The plots clearly show an exponential decay. Regarding the displacements in the longitudinal modes, there are edge effects, which disappear if the particle that is excited is at the center of the chain. In principle this cannot be realized in experi- 


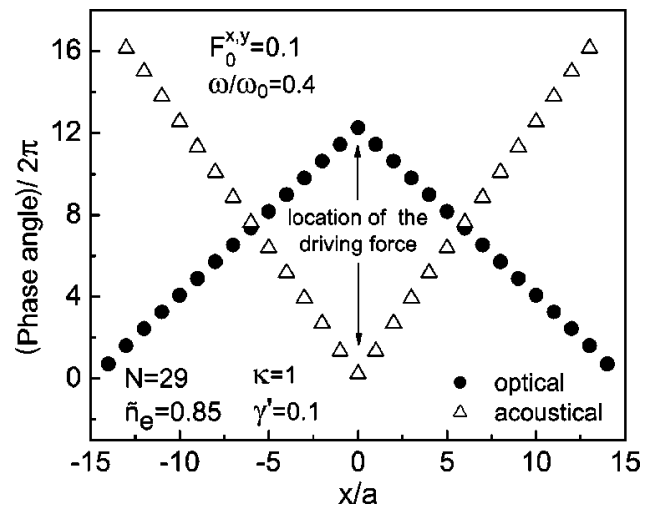

FIG. 6. Phase angle as a function of the distance from the location of the driving force. The calculations are done for a system of $N=29$ particles. Optical and longitudinal waves both propagate away from the excitation region; they are backward and forward, respectively.

ments; however, except for the first two particles the amplitudes of the displacements have the same damping rate and the same phase angles whether the excited particle is the one at the end of the chain or the one in the middle. This is why for all the calculations we have considered a force $F_{0}^{x} e^{-i \omega t} \delta_{l, N / 2}$ instead of $F_{0}^{x} e^{-i \omega t} \delta_{l, N}$ in Eq. (17).

Fitting the amplitudes to an exponential curve yields $k_{i}$. In order to find the dispersion of $k_{r}$, we calculated the wave's phase $\phi$ as a function of the position and fitted it to a straight line. The definition of phase velocity, as being the ratio between the frequency and the wave number, indeed yields $k_{r} a=\Delta \phi$. In Fig. 6 the phase angle as a function of the distance is plotted. It is interesting to observe that $k_{r}$ and $k_{i}$ are independent of the intensity of the driving force $F_{0}$, as expected in a harmonic model, and the results do not change if instead of $F_{0} e^{-i \omega t}$, which is a complex force, we consider a real force $I_{0} \sin \omega t$, as in the experiment; what actually plays a central role is just the driving frequency. Optical and longitudinal waves both propagate away from the excitation region; they are backward and forward, respectively. It should be noticed that in a 1D chain with finite length one should expect that only standing waves would be allowed; the effect of gas damping is the suppression of the reflected wave from the chain's end.

It is interesting to observe that for low densities the two calculational methods, that is, the one in which the driving force is neglected and the one in which it is explicitly taken into account, give the same results for the dispersion curves, when only first neighbor interactions are included. In Fig. 7 the results of the two approaches for the optical branch are compared. Note that outside the bond defined by the two dotted horizontal lines the phonon mode is strongly damped.

Another remarkable effect that reflects the anisotropy of the system is observed with increasing density: the profile of displacements for the longitudinal mode is no longer a pure damped exponential [see Fig. 8(a)], because reflected waves from the chain's end start to appear, while the amplitudes for the transverse mode are still exponentially decaying. We can infer that the effect of damping is not due simply to friction, but also to the external confining potential. This is confirmed

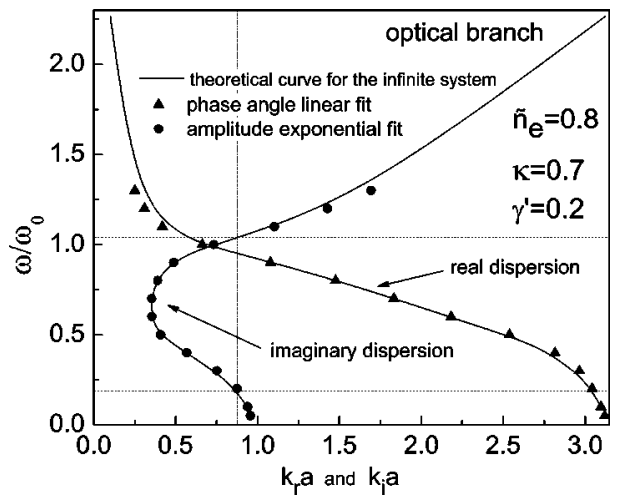

FIG. 7. Comparison between the standard calculation, in which the external driving force is neglected, and the "exact" one, in which the external driving force is explicitly considered. The region on the left of the vertical dotted curve is the region in which the condition $k_{i} a \leqslant \kappa / \widetilde{n}_{e}$ is satisfied; the region between the horizontal dotted curves is the region in which the sums are convergent. Note that the two approaches give the same results in the region of convergence.
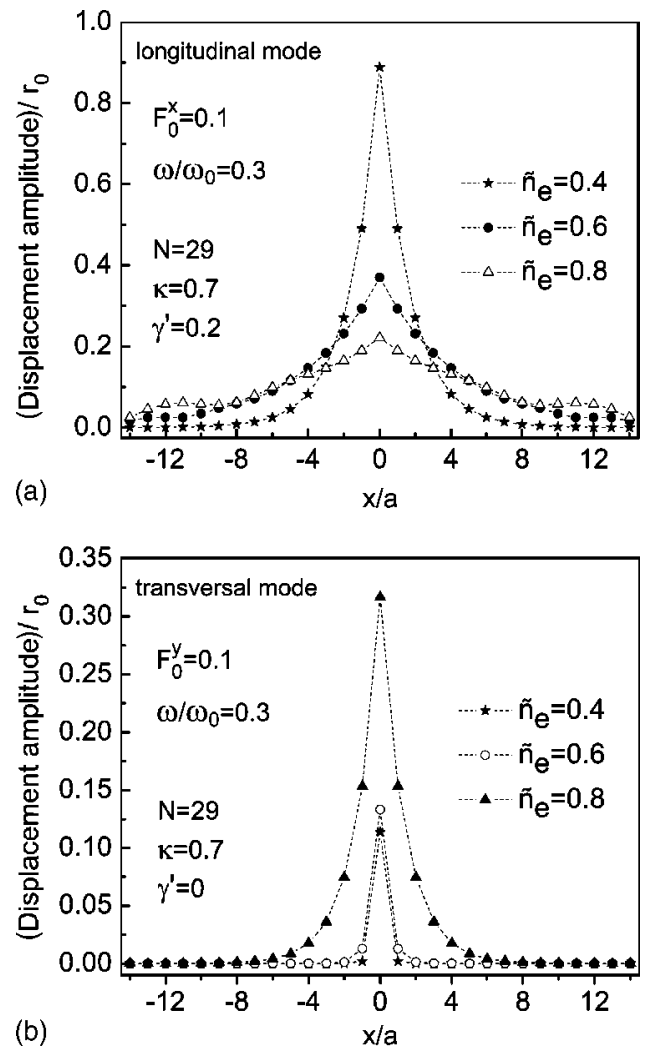

FIG. 8. (a) Profile of the amplitudes of displacements in the longitudinal mode as a function of the density. For high densities the profile is no longer a simply decaying exponential. (b) Profile of the amplitudes of displacements in the transverse mode as a function of the density in the case without friction. Even in the absence of friction the profile is still a decaying exponential. 

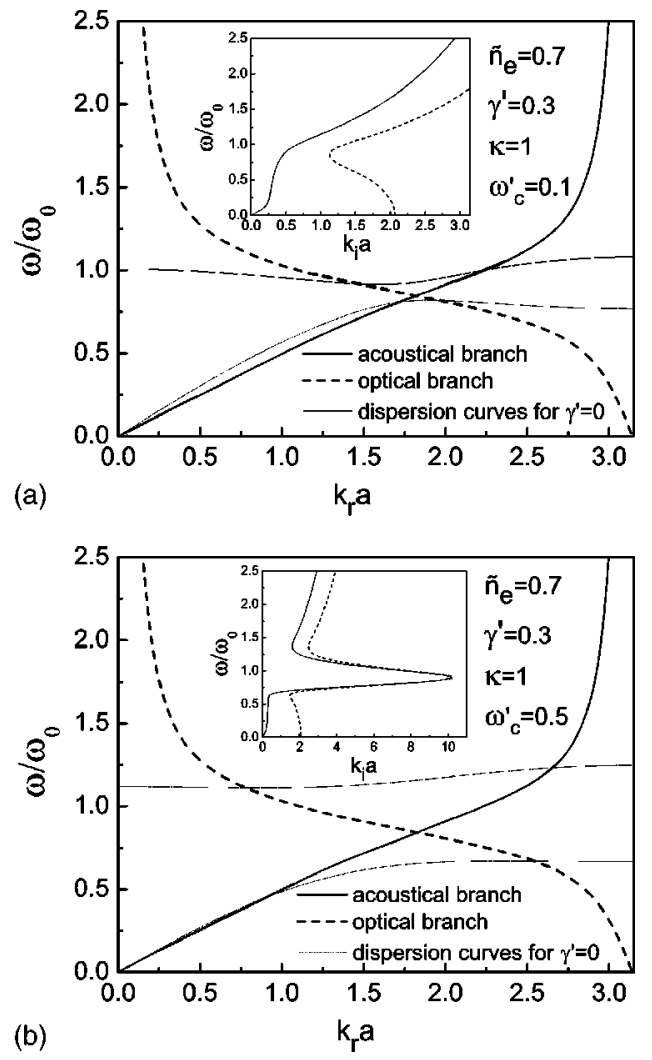

FIG. 9. (a) Real part of the dispersion relations for a weak perpendicular magnetic field; (b) real part of the dispersion relations for a strong perpendicular magnetic field. The insets show the imaginary part of the dispersion relations.

by the calculation of the amplitude profile when $\gamma^{\prime}=0$, which is reported in Fig. 8(b): even in the absence of the friction and in the case that the driving frequency is low enough, an exponential decay of amplitudes with distance is still found.

When we include a perpendicular magnetic field, a coupling is introduced between motion along the $x$ and along $y$ direction. Apart from increased mathematical complexity, the scheme developed before is still valid. It is possible to infer the real part of the dispersion relations from the phase angles and the imaginary part from the amplitude of displacements. In the absence of friction and driving force, the optical and acoustical branches are confined in different frequency bands [see thin solid curves in Figs. 9(a) and 9(b)], which do not cross each other and have a prohibited gap [18]. The optical frequencies follow the cyclotron frequency and for very high field strength there is no significant difference between $\omega_{\text {opt }}$ and $\omega_{c}$. The acoustical frequencies, on the other hand, decrease with increasing magnetic field strength. The gap between the optical branch and the acoustical one for large magnetic field approaches $\omega_{c}$.

In the presence of friction and driving force, there are drastic changes in the dispersion relations. The frequencies are no longer confined in different bands, because the frequency of oscillation is that of the external force, which can be varied continously. The results of the calculations for different intensities of the magnetic field are shown in Figs. 9(a)
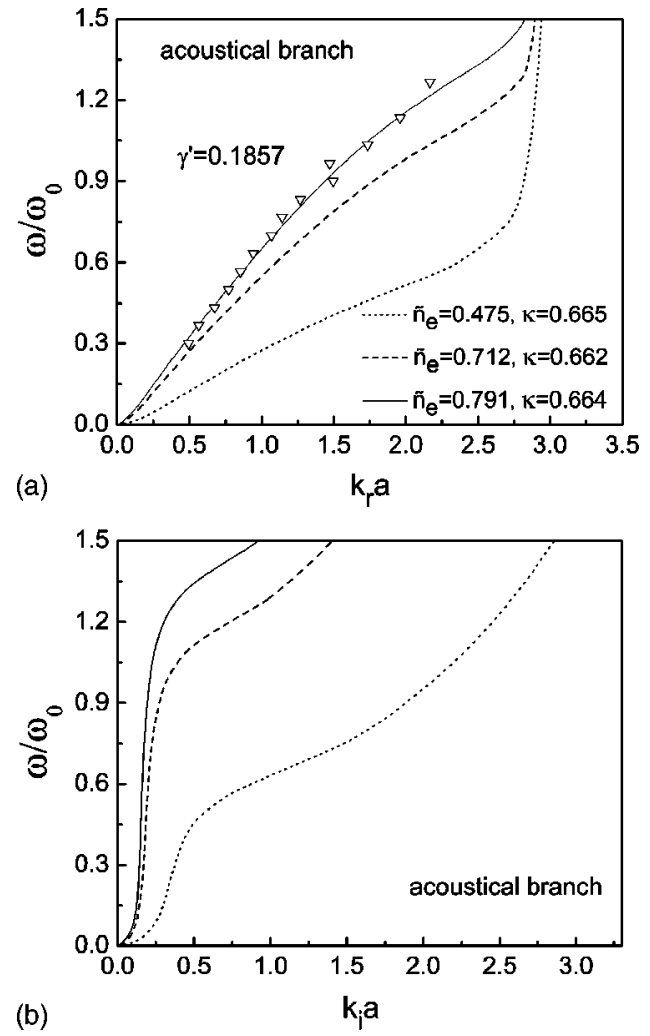

FIG. 10. Theoretical (curves) dispersion relations for the acoustical mode and comparison with experimental data (symbols) from Ref. [7] for the one-chain structure: (a) real part of the acoustical branch; (b) imaginary part of the acoustical branch.

and 9(b). There are no significant differences in the behavior of the real part of the dispersion relations with respect to the case without magnetic field. The imaginary dispersion relations clearly show that the waves are overdamped in the band gap, a region where large values for $k_{i} a$ are found. Notice that friction reduces the slope of the acoustical branch in the small $k_{r} a$ region. When the curve enters the gap region it becomes strongly damped as is clearly seen from the insets of Figs. 9(a) and 9(b). The optical mode is more strongly damped for all frequencies than the acoustical one. Furthermore, the dispersion of the optical branch is strongly modified by friction, i.e., it attains a negative dispersion for all values of the frequency.

\section{COMPARISON WITH EXPERIMENT}

In Figs. 10 and 11 the real and imaginary dispersion relations for the acoustical and optical modes for the single chain configuration are presented, respectively, for different values of the parameters.

The calculated dispersion relations are compared with the experimental data of Ref. [7]. The experimental data are in good agreement with the theoretical calculations, although the system realized in the laboratory is slightly different from the one investigated in the theory. As a matter of fact, in the experiment the interparticle spacing was not uniform: it was $15 \%$ smaller in the center than at the chain's end. Due to the 

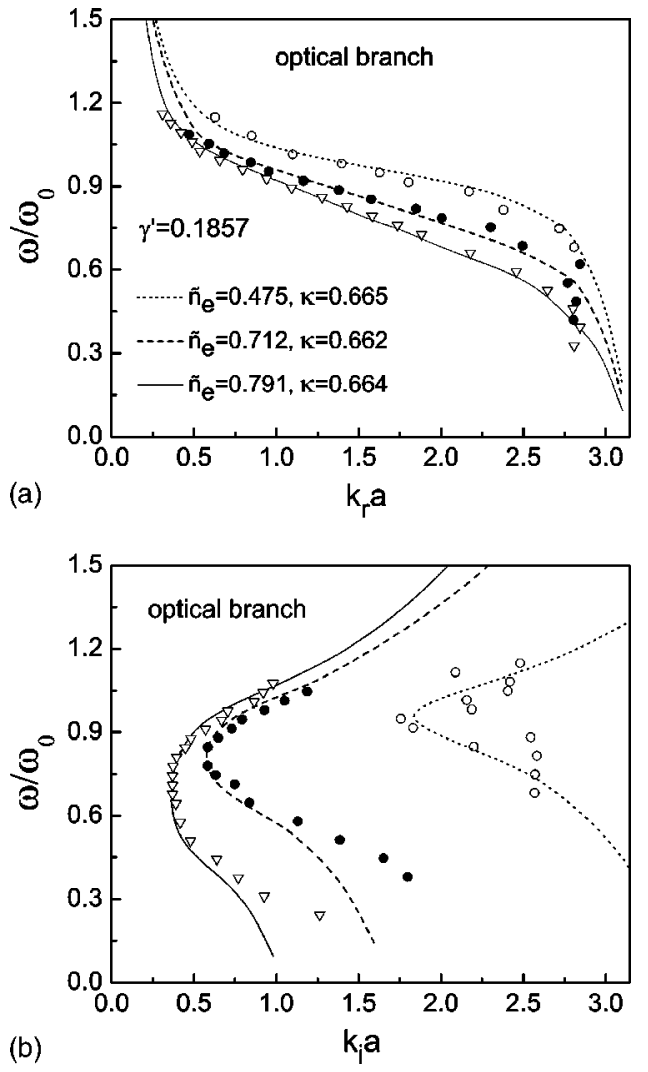

FIG. 11. The same as Fig. 10 but now for the optical branch.

strong damping this density gradient is not very important in the forced oscillation considered. As in the case without friction, the optical mode has negative dispersion, while the longitudinal one has positive dispersion. The dispersion depends on density and therefore on interparticle spacing. For the acoustical mode the frequencies of vibrations increase with decreasing interparticle distance, while for optical vibration the frequencies decrease with increasing densities. Furthermore, for low $\widetilde{n}_{e}$ the exponential decay is stronger in both cases, which implies a highly damped wave. These findings can be easily explained because for smaller interparticle distance the interaction forces are larger, or in other words for low densities the interaction between the particles is rather weak and consequently the effect of a local perturbation is less disruptive for the other particles. From Fig. 11(b) it is seen that the optical mode is mostly constrained to a central frequency band. Comparing the optical branches in the absence of friction (see Fig. 7 of Ref. [18]) with the one in the presence of gas damping, it is observed that with damping the wave propagates beyond the frequency band allowed in the absence of damping. For $k_{r} a=\pi, \omega_{\text {opt }}$ is always equal to zero when $\gamma^{\prime} \neq 0$, independent of the experimental parameters. This means that in the presence of damping, the softening of the optical mode no longer signals a structural phase transition from a single chain structure to a double chain structure.

It is, however, interesting to study the behavior of the dispersion relations when the density approaches the critical value for which the continuous structural transition from the
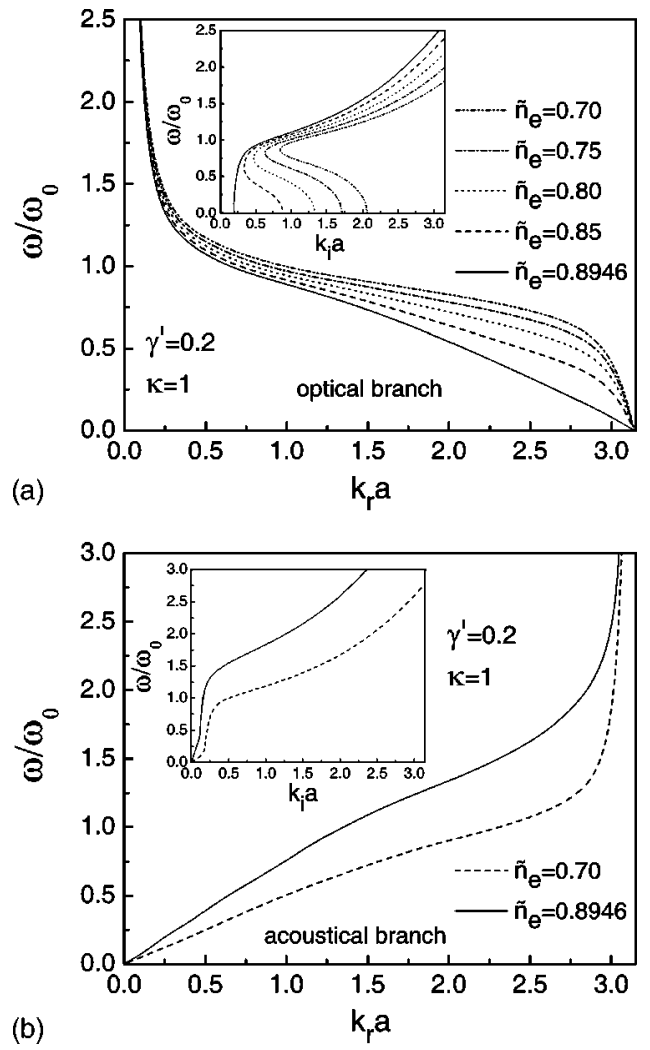

FIG. 12. Dispersion relations for different values of the density approaching the critical values for which the zig-zag transition from 1 chain structure to 2 chain structure is observed: (a) optical branches; (b) acoustical branches. In the insets the imaginary parts of the dispersion relations are plotted.

one-chain to the two-chain structure occurs. The results are shown in Fig. 12.

The optical branch softens when approaching the critical density, while the acoustical branch is hardened. Notice that at the phase transition point (i) the real part of the optical dispersion becomes linear for $k_{r} a>1.5$, (ii) there is a drastic change of slope in the optical imaginary dispersion, and (iii) the optical mode becomes less damped. The real and imaginary acoustical dispersions are less strongly influenced near the zigzag transition. This can be easily explained by the fact that the zigzag transition, which is responsible for the splitting of the chain, acts in the $y$ direction. Therefore, signature of the zigzag transition are more easily detected in the optical phonon mode.

\section{FORCED OSCILLATIONS IN A MULTICHAIN STRUCTURE}

In Figs. 13 and 14(a) the real and imaginary parts of the dispersion relations for the forced oscillations of the twoand three- chain configurations are reported, respectively. We used the approach given in the first part of Sec. IV. Therefore, the dispersion relations in Figs. 13 and 14 are given only in that part of the Brillouin zone where the sums in Eqs. 14(a) and 14(b) are convergent. 

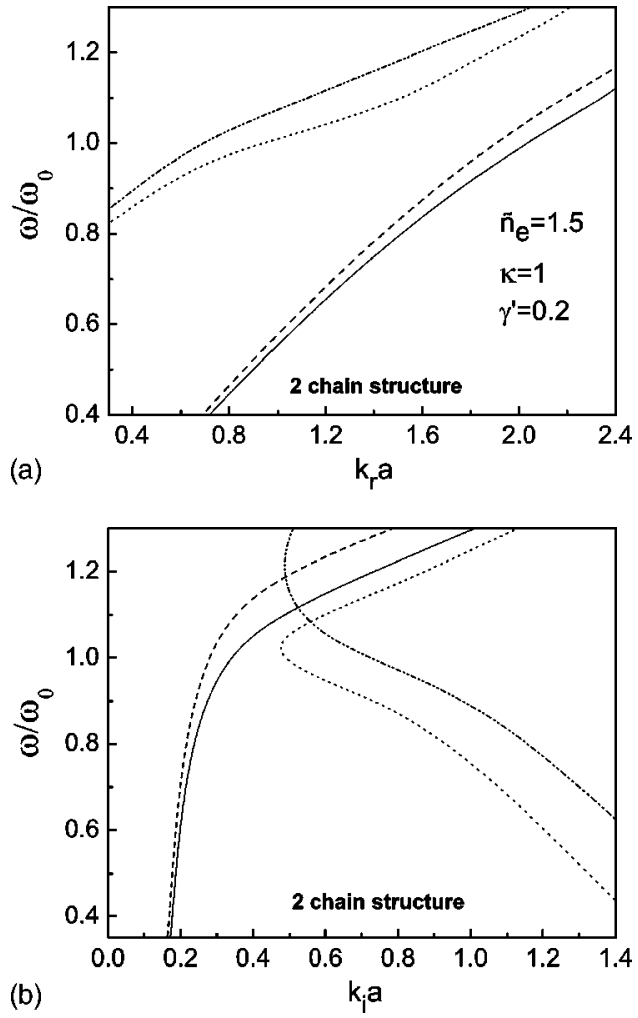

FIG. 13. Theoretical dispersion relations for the two-chain structure: (a) real part; (b) imaginary part.
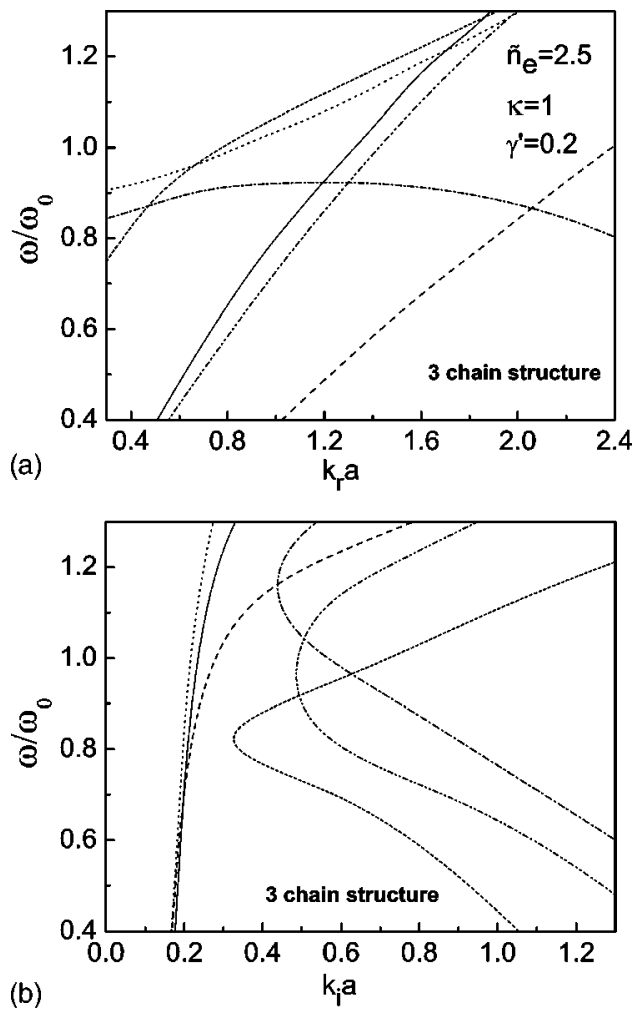

FIG. 14. Theoretical dispersion relations for the three-chain structure: (a) real part; (b) imaginary part.
From Fig. 13(a) and 14(a) it is evident that there is a remarkable difference in the optical branches between the single chain and the two- and three-chain structures. In the first case the optical mode has negative dispersion as stated before, while for the two- and three- chain structures the optical frequencies do not exhibit a monotonic behavior. This feature can be attributed to the fact that for the single chain configuration in the case of the transverse mode the restoring force is only due to the parabolic confining potential, while in the multichain configuration the restoring force depends both on the external confinement and on the particle repulsion.

Figures 13(b) and 14(b) exhibit some similarities with the single chain case: (i) for the acoustical modes the damping is an increasing function of the driving frequency, (ii) the optical modes are mostly constrained to a frequency band, and (iii) the optical modes are more strongly damped. The approach used for the calculation of the dispersion relations for the multichain is the same considered in Sec. IV for an infinite number of particles, this is why in Figs. 13 and 14 the dispersion relations are not presented in the whole first Brillouin zone, but only in that range of the frequency corresponding to convergent sums.

\section{CONCLUSION}

The ground state and the normal modes of a Q1D multichain system can be studied experimentally in a dusty plasma, where micrometer-sized particles are externally confined by electric fields in the sheath above the lower electrode. The sheath conforms to the shape of the electrode, so building up an electrode with a groove-shaped depression in one direction allows the realization of a parabolic confining potential and, as a consequence, the formation of a chainlike crystal in that direction.

We investigated the structural properties and the normal modes of such a classical Q1D system of particles interacting through a Yukawa-type potential. The structural transitions are of first (primarily) and second order. The normal modes of the system were calculated first, neglecting the effects of dissipation induced by gas drag and then considering explicitly the presence of friction. The normal modes consist of longitudinal (acoustical modes) and transversal (optical modes). The number of acoustical branches is equal to the number of optical branches and is equal to the number of chains in the system. In the presence of friction, the free oscillations of the system are exponentially damped in time. The effect of a constant magnetic field on the dispersion relations was investigated and we found that the acoustical and optical branches no longer cross.

Particular attention was paid to the case of forced oscillations induced by an external driving force, as was investigated in the experiments. We found that earlier approaches to calculating the phonon dispersion relations are no longer valid. Our theoretical results were compared with experimental data and a remarkably good agreement between theory and experiment was found. 
Finally, we made predictions for the single chain dispersion relations in the presence of a perpendicular magnetic field and for the multichain dispersion relations when the modes are excited by an external driving force. We found some substantial differences as well as some similarities in the dispersion relations between the single and multichain structures.

\section{ACKNOWLEDGMENTS}

This work was supported in part by the European Community's Human Potential Program under Contract HPRNCT-2000-00157 "Surface Electrons," the Flemish Science Foundation (FWO-Vl), Belgian Science Policy, the GOA, and the visitors program of the Max Planck Institute. We are very grateful to Professor J. Goree for providing us with the experimental data on the transverse mode of the single chain structure.

\section{APPENDIX A}

The equations of motion for particles in the three-chain configuration in the presence of friction are in matrix form:

$$
\begin{aligned}
& \left(\begin{array}{l}
\ddot{x}_{n}^{\prime(1)} \\
\ddot{y}_{n}^{\prime(1)} \\
\ddot{x}_{n}^{\prime(2)} \\
\ddot{y}_{n}^{\prime(2)} \\
\ddot{x}_{n}^{\prime(3)} \\
\ddot{y}_{n}^{\prime(3)}
\end{array}\right)=\gamma^{\prime}\left(\begin{array}{c}
\dot{x}_{n}^{\prime(1)} \\
\dot{y}_{n}^{\prime(1)} \\
\dot{x}_{n}^{\prime(2)} \\
\dot{y}_{n}^{\prime(2)} \\
\dot{x}_{n}^{\prime(3)} \\
\dot{y}_{n}^{\prime(3)}
\end{array}\right) \\
& +\left(\begin{array}{cccccc}
-B_{1} & 0 & -B_{3} & 0 & -B_{5} & 0 \\
0 & -B_{2} & 0 & -B_{4} & 0 & -B_{6} \\
-B_{3} & 0 & -B_{1} & 0 & -B_{3} & 0 \\
0 & -B_{4} & 0 & -B_{2} & 0 & -B_{4} \\
-B_{5} & 0 & -B_{3} & 0 & -B_{1} & 0 \\
0 & -B_{6} & 0 & -B_{4} & 0 & -B_{2}
\end{array}\right) \\
& \times\left(\begin{array}{c}
x_{j}^{\prime(1)} \\
y_{j}^{\prime(1)} \\
x_{j}^{\prime(2)} \\
y_{j}^{\prime(2)} \\
x_{j}^{\prime(3)} \\
y_{j}^{\prime(3)}
\end{array}\right)
\end{aligned}
$$

where the superscript labels the row in which the particle is placed. The coefficients are

$$
\begin{gathered}
B_{1}=\frac{\tilde{n}_{e}^{3}}{54} \sum_{j} \frac{e^{-3 j \kappa / \tilde{n}_{e}}}{j^{3}}\left[2+6 \frac{j \kappa}{\tilde{n}_{e}}+9 \frac{j^{2} \kappa^{2}}{\tilde{n}_{e}^{2}}\right], \\
B_{2}=-\frac{\tilde{n}_{e}^{3}}{54} \sum_{j} \frac{e^{-3 j \kappa / \tilde{n}_{e}}}{j^{3}}\left(1+3 \frac{j \kappa}{\tilde{n}_{e}}\right),
\end{gathered}
$$

$$
\begin{aligned}
B_{3}= & \frac{\tilde{n}_{e}^{3}}{54} \sum_{j} \frac{e^{-3 \kappa r_{12} / \tilde{n}_{e}}}{r_{12}^{5}}\left[\left(j+\frac{1}{2}\right)^{2}\left(\frac{9 \kappa r_{12}}{\tilde{n}_{e}}+\frac{9 \kappa^{2} r_{12}^{2}}{\tilde{n}_{e}^{2}}+3\right)\right. \\
& \left.-r_{12}^{2}\left(1+\frac{3 \kappa r_{12}}{\tilde{n}_{e}}\right)\right], \\
B_{4}= & \frac{\tilde{n}_{e}^{3}}{54} \sum_{j} \frac{e^{-3 \kappa r_{12} / \tilde{n}_{e}}}{r_{12}^{5}}\left[c_{3}^{2}\left(\frac{9 \kappa r_{12}}{\tilde{n}_{e}}+\frac{9 \kappa^{2} r_{12}^{2}}{\tilde{n}_{e}^{2}}+3\right)\right. \\
& \left.-r_{12}^{2}\left(1+\frac{3 \kappa r_{12}}{\tilde{n}_{e}}\right)\right], \quad \\
B_{5}= & \frac{\tilde{n}_{e}^{3}}{54} \sum_{j} \frac{e^{-3 \kappa r_{13} / \tilde{n}_{e}}}{r_{13}^{3}}\left(\frac{9 \kappa r_{13}}{\tilde{n}_{e}}+\frac{9 \kappa^{2} r_{13}^{2}}{\tilde{n}_{e}^{2}}+3\right), \\
B_{6}= & \frac{\tilde{n}_{e}^{3}}{54} \sum_{j} \frac{e^{-3 \kappa r_{13} / \tilde{n}_{e}}}{r_{13}^{5}}\left[4 c_{3}^{2}\left(\frac{9 \kappa r_{13}}{\tilde{n}_{e}}+\frac{9 \kappa^{2} r_{13}^{2}}{\tilde{n}_{e}^{2}}+3\right)\right. \\
& \left.-r_{13}^{2}\left(1+\frac{3 \kappa r_{13}}{\tilde{n}_{e}}\right)\right],
\end{aligned}
$$

where $r_{12}=\sqrt{(j+1 / 2)^{2}+c_{3}^{2}}$ and $r_{13}=\sqrt{j^{2}+4 c_{3}^{2}}$.

The equations of motion for the two-chain structure can be obtained by the $4 \times 4$ submatrices which are included in the top left part of the matrix in Eq. (A1): the coefficients involved in this case are $B_{1}, B_{2}, B_{3}$, and $B_{4}$, with the substitution $\tilde{n}_{e} / 3 \rightarrow \tilde{n}_{e} / 2$.

In the presence of a constant magnetic field $\vec{B}=(0,0, B)$ the equations of motions for the two- and three-chain structures are easily obtained from the equations of motion with $B=0$, adding the coupling terms $\dot{y}_{n}^{\prime(i)} \omega_{c}^{\prime}$ and $-\dot{x}_{n}^{(i)} \omega_{c}^{\prime}$ to the equations for $x$ and $y$ motion, respectively, for particles sitting in the $i$ th row.

Obviously, the case without gas drag is immediately recovered by setting $\gamma^{\prime}=0$.

\section{APPENDIX B}

The eigenfrequencies in the three-chain configuration are:

$$
\begin{gathered}
\omega_{a c}^{(1)}=\sqrt{B_{1}-B_{5}-\gamma^{2} / 4}-i \gamma / 2, \\
\omega_{o p t}^{(1)}=\sqrt{1+B_{2}-B_{6}-\gamma^{2} / 4}-i \gamma / 2, \\
\omega_{a c}^{(2)}=\sqrt{B_{1}+B_{5} / 2+\sqrt{B_{5}^{2}+8 B_{3}^{2}} / 2-\gamma^{2} / 4}-i \gamma / 2, \\
\omega_{o p t}^{(2)}=\sqrt{1+B_{2}+B_{6} / 2+\sqrt{B_{6}^{2}+8 B_{4}^{2}} / 2-\gamma^{2} / 4}-i \gamma / 2, \\
\omega_{a c}^{(3)}=\sqrt{B_{1}+B_{5} / 2-\sqrt{B_{5}^{2}+8 B_{3}^{2}} / 2-\gamma^{2} / 4}-i \gamma / 2, \\
\omega_{o p t}^{(3)}=\sqrt{1+B_{2}+B_{6} / 2-\sqrt{B_{6}^{2}+8 B_{4}^{2}} / 2-\gamma^{2} / 4}-i \gamma / 2,
\end{gathered}
$$

where the coefficients $B_{n}$ are the same as in Appendix A. In the two-chain configuration the eigenfrequencies are

$$
\omega_{a c}^{(1)}=\sqrt{B_{1}+B_{3}-\gamma^{2} / 4}-i \gamma / 2,
$$




$$
\begin{gathered}
\omega_{o p t}^{(1)}=\sqrt{B_{2}+B_{4}-\gamma^{2} / 4}-i \gamma / 2, \\
\omega_{a c}^{(2)}=\sqrt{1+B_{1}-B_{3}-\gamma^{2} / 4}-i \gamma / 2, \\
\omega_{o p t}^{(2)}=\sqrt{1+B_{2}-B_{4}-\gamma^{2} / 4}-i \gamma / 2 .
\end{gathered}
$$

In this case the coefficients $B_{n}$ are obtained from the coefficients in Appendix A with the substitution $\tilde{n}_{e} / 3 \rightarrow \tilde{n}_{e} / 2$.

\section{APPENDIX C}

We present the analytical expressions for the displacements $A_{l}^{x, y}$ calculated from the Kramer's rule [Eq. (42)] in the case of a system of $N=29$ particles. The central particle at which the driving force is acting is labeled with $N=0$. For reasons of symmetry $A_{-l}^{x, y}=A_{l}^{x, y}$ :

$$
\begin{aligned}
A_{0}^{x, y}= & \left(a^{2}-b^{2}\right)\left(-b^{2}-b a+a^{2}\right)\left(-b^{2}+b a+a^{2}\right)\left(b^{4}+4 b^{3} a\right. \\
- & \left.4 b^{2} a^{2}-b a^{3}+a^{4}\right)\left(b^{4}-4 b^{3} a-4 b^{2} a^{2}+b a^{3}+a^{4}\right) C / D, \\
A_{1}^{x, y}= & -a b\left(-b^{3}-2 b^{2} a+b a^{2}+a^{3}\left(b^{3}-2 b^{2} a-b a^{2}+a^{3}\right)\right. \\
& \times\left(-7 b^{6}+14 b^{4} a^{2}-7 b^{2} a^{4}+a^{6}\right) C / D, \\
A_{2}^{x, y}= & a b^{2}\left(-a^{6}+a^{5} b+5 a^{4} b^{2}-4 a^{3} b^{3}-6 a^{2} b^{4}+3 a b^{5}+b^{6}\right) \\
& \times\left(-a^{6}-a^{5} b+5 a^{4} b^{2}+4 a^{3} b^{3}-6 a^{2} b^{4}-3 a b^{5}\right. \\
& \left.+b^{6}\right) C / D, \\
& A_{3}^{x, y}=-a b^{3}\left(-3 b^{2}+a^{2}\right)\left(a^{2}-b^{2}\right)\left(-2 b^{2}+a^{2}\right) \\
& \times\left(b^{4}-4 b^{2} a^{2}+a^{4}\right) C / D,
\end{aligned}
$$

$$
\begin{aligned}
& A_{4}^{x, y}=-b^{4}\left(-a^{5}+a^{4} b+4 a^{3} b^{2}-3 a^{2} b^{3}-3 a b^{4}+b^{5}\right) \\
& \times\left(a^{5}+a^{4} b-4 a^{3} b^{2}-3 a^{2} b^{3}+3 a b^{4}+b^{5}\right) C / D, \\
& A_{5}^{x, y}=-a b^{5}\left(-a^{2}-b a+b^{2}\right)\left(-a^{2}+b a+b^{2}\right) \\
& \times\left(a^{4}-5 a^{2} b^{2}+5 b^{4}\right) C / D \text {, } \\
& A_{6}^{x, y}=b^{6}\left(a^{2}-b^{2}\right)\left(-b^{3}-3 b^{2} a+a^{3}\right)\left(b^{3}-3 b^{2} a+a^{3}\right) C / D, \\
& A_{7}^{x, y}=-a b^{7}\left(-2 b^{2}+a^{2}\right)\left(2 b^{4}-4 b^{2} a^{2}+a^{4}\right) C / D, \\
& A_{8}^{x, y}=b^{8}\left(b^{3}-2 b^{2} a-b a^{2}+a^{3}\right)\left(-b^{3}-2 b^{2} a+b a^{2}+a^{3}\right) C / D \text {, } \\
& A_{9}^{x, y}=-a b^{9}\left(-3 b^{2}+a^{2}\right)\left(a^{2}-b^{2}\right) C / D, \\
& A_{10}^{x, y}=b^{10}\left(-b^{2}+a b+a^{2}\right)\left(-b^{2}-a b+a^{2}\right) C / D, \\
& A_{11}^{x, y}=a b^{11}\left(2 b^{2}-a^{2}\right) C / D, \\
& A_{12}^{x, y}=b^{12}\left(a^{2}-b^{2}\right) C / D \\
& A_{13}^{x, y}=-a b^{13} C / D, \\
& A_{14}^{x, y}=-b^{14} / D \text {, } \\
& \text { where } \quad D=a\left(-3 b^{2}+a^{2}\right)\left(5 b^{4}-5 b^{2} a^{2}+a^{4}\right)\left(b^{8}-8 b^{6} a^{2}+14 b^{4} a^{4}\right. \\
& \left.-7 b^{2} a^{6}+a^{8}\right) \text {. }
\end{aligned}
$$

[1] J. H. Chu and Lin I, Phys. Rev. Lett. 72, 4009 (1994).

[2] H. Thomas, G. E. Morfill, V. Demmel, J. Goree, B. Feuerbacher, and D. Möhlmann, Phys. Rev. Lett. 73, 652 (1994).

[3] Y. Hayashi and K. Tachibana, Jpn. J. Appl. Phys., Part 2 33, L804 (1994).

[4] A. Melzer, T. Trottenberg, and A. Piel, Phys. Lett. A 191, 301 (1994).

[5] E. Wigner, Phys. Rev. 46, 1002 (1934).

[6] H. Ikezi, Phys. Fluids 26, 1764 (1986).

[7] B. Liu, K. Avinash, and J. Goree, Phys. Rev. Lett. 91, 255003 (2003).

[8] A. Homann, A. Melzer, S. Peters, and A. Piel, Phys. Rev. E 56, 7138 (1997).

[9] S. A. Tatarkova, A. E. Carruthers, and K. Dholakia, Phys. Rev. Lett. 89, 283901 (2002).

[10] G. Birkl, S. Kassner, and H. Walther, Nature (London) 357, 310 (1992).

[11] P. Glasson, V. Dotsenko, P. Fozooni, M. J. Lea, W. Bailey, G. Papageorgiou, S. E. Andresen, and A. Kristensen, Phys. Rev. Lett. 87, 176802 (2001).

[12] J. I. Cirac and P. Zoller, Phys. Rev. Lett. 74, 4091 (1995).

[13] P. M. Platzman and M. I. Dykman, Science 284, 1967 (1999).

[14] A. Brown, Can. J. Chem. 52, 791 (1974).
[15] P. Segovia, D. Purdie, M. Hengsberger, and Y. Baer, Nature (London) 402, 504 (1999).

[16] M. T. Cvitas and A. Siber, Phys. Rev. B 67, 193401 (2003).

[17] A. Siber, Phys. Rev. B 66, 235414 (2002).

[18] G. Piacente, I. V. Schweigert, J. J. Betouras, and F. M. Peeters, Solid State Commun. 128, 57 (2003); Phys. Rev. B 69, 045324 (2004).

[19] J. Goree (private communication).

[20] U. Konopka, G. E. Morfill, and L. Ratke, Phys. Rev. Lett. 84, 891 (2000).

[21] L. Radzihovsky, E. Frey, and D. R. Nelson, Phys. Rev. E 63, 031503 (2001).

[22] L. Candidoŏ, J. P. Rino, N. Studart, and F. M. Peeters, J. Phys.: Condens. Matter 10, 11627 (1998).

[23] N. W. Ashcroft and N. D. Mermin, Solid State Physics (Holt Saunders, Philadelphia, 1976).

[24] J. H. Van Leeuwen, J. Phys. Radium 6, 361 (1921).

[25] A. V. Chaplik, Sov. Phys. JETP 35, 395 (1972).

[26] L. Bonsall and A. A. Maradudin, Phys. Rev. B 15, 1959 (1977)

[27] X. Wang, A. Bhattacharjee, and S. Hu, Phys. Rev. Lett. 86, 2569 (2001).

[28] M. Rosenberg and G. Kalman, Phys. Rev. E 56, 7166 (1997). 
[29] J. B. Pieper and J. Goree, Phys. Rev. Lett. 77, 3137 (1996).

[30] A. Homann, A. Melzer, R. Madani, and A. Piel, Phys. Lett. A 242, 173 (1998).

[31] S. Nunomura, D. Samsonov, and J. Goree, Phys. Rev. Lett. 84, 5141 (2000).

[32] S. V. Vladimirov, P. V. Shevchenko, and N. F. Cramer, Phys. Rev. E 56, R74 (1997).
[33] B. Liu, J. Goree, V. Nosenko, and L. Boufendi, Phys. Plasmas 10, 9 (2003).

[34] H. Goldstein, Classical Mechanics (Narosa Publishing House, New Delhi, PA, 1985).

[35] T. Misawa, N. Ohno, K. Asano, M. Sawai, S. Takamura, and P. K. Kaw, Phys. Rev. Lett. 86, 1219 (2001). 